\title{
The Simien Fox in the Balé Mountains
}

\author{
P. A. Morris \\ and \\ J. R. Malcolm
}

An endemic species in Ethiopia, the Simien fox survives in only four areas, and is in danger of extinction in three of them, including the mountains from which it is named. Its only predator is man, and the chief causes of its decline are habitat destruction and the unfounded belief that it takes domestic stock. The Balé Mountains now hold the largest Simien fox population, which the authors estimate at between 350 and 475 animals. The urgent need is for the Balé Mountains National Park, proposed in 1970, to be gazetted.

The Simien fox Canus simensis is a distinctive and very localised endemic carnivore found only on certain mountains in Ethiopia. Standing $60 \mathrm{~cm}$ at the shoulder it looks like a foxy-red coyote and is larger than the jackals to which it is probably most closely related; ${ }^{5}$ virtually nothing is known of its ecology or social organisation. Of the four known populations three are in imminent danger of extinction, including that in the Simien Mountains. Our observations were made on the southern race in the Bale mountains between 1971 and 1977. P. A. M. spent three weeks there in December 1971 and January 1972 , and five days in April 1975, observing Simien foxes in the course of a general survey of the local fauna. J. R. M. spent six weeks there in January and February 1976, specifically studying the distribution and habits of Simien foxes, and four weeks in December 1976-January 1977.

\section{Distribution and Status}

The Simien fox was first described by Rüppell in 1835 , and has been regularly observed in its type locality since, although by 1938 it had decreased sufficiently to need protection. ${ }^{6}$ This northern race is now listed as 'endangered' 7 and is rarely seen. ${ }^{2}$ A 1974 report suggested that only half-a-dozen remained in the Simien National Park ${ }^{1}$, and in January 1976, the park warden estimated that no more than 20 survived there (H. Hurni, pers. comm.). On the 
be spread evenly over their foraging areas. Other parts of the park produced 99 sightings. The density of fox faeces in this study area, the number of fox excavations, and the availability of suitable rodent prey were noted as indices of abundance, and compared with the comparable figures for both the whole park and the area where fox density was known from direct sightings. A distribution map of the proposed park was then produced with rough estimates of fox densities in different areas. Our estimated total was about 350 foxes.

They were found to be relatively numerous in all major grassland regions that support large rodent populations; uncommon in the large area of Erica and Phillipia scrub covering the slopes up to $3800 \mathrm{~m}$.; and were not seen at all in the Hagenia/Hypericum forests of lower altitudes. The highest densities (perhaps 2 per square kilometre) occur on the Sanetti plateau, on the eastern side, some $20 \mathrm{~km}$ south-west of Goba, at about $4000 \mathrm{~m}$. About 25 inhabit the grasslands along the Ueb Valley in the west of the park, and a few occur in the grasslands around Gaysay Mountain, west of Dinshu (Gurie on some maps). Probably very few survive in the Bale Mountains outside this area.

J.R.M., aided by a grant from the FPS, spent from December 20th 1976 to January 20 th 1977 in the study area and also in a small new area, and reported that numbers may even have increased. He now thought that our estimate of 350 was the minimum figure, with up to 475 as the maximum.

\section{Daily Activity}

Simien foxes in the Bale Mountains are primarily diurnal, but there was some evidence of activity at night, especially in moonlight. Noisy greetings were heard at 20.30 and 20.40 and five foxes that J.R.M. observed were still active when darkness fell. Of eight foxes still lying quietly at last light, three had moved before dawn at 06.15. In the Simien Mountains the few remaining foxes are reported to be almost entirely nocturnal, probably due to human disturbance. In January and February, despite night temperatures as low as $-7^{\circ} \mathrm{C}$, foxes made little attempt to find shelter. Three of the 8 seen sleeping appeared to have selected areas of slightly longer grass (about $0.5 \mathrm{~m}$ ) in which to lie, others had curled up in the open. Droppings in small caves and rock fissures suggest that these are sometimes used, possibly in the rainy season (April-November). No fox burrows were found despite careful searches in suitable habitat.

During daylight hours activity fell into three periods. Between 06.00-09.00 and 17.00-18.30, around dawn and dusk, the majority of foxes (68 per cent) were in groups; little foraging was observed and greetings were common. The groups seemed to disperse between 08.30 and 09.00 when the common diurnal rodents became active. Two foxes that did not join groups were observed resting quietly until around 08.30 before beginning to hunt. In the middle of the day foxes were usually solitary, only 14 per cent (16/111) seen by J.R.M. between 09.00 and 17.00 being in groups. Following foxes for $12 \frac{1}{2}$ hours in the middle of the day revealed that they rested for 60 per cent of the time; in January and February few seemed to spend more than four or five hours a day foraging. During resting periods, which sometimes exceeded two hours and were spread evenly through the day, they would lift their heads every 5-10 minutes to look around. 


\section{Foraging Behaviour}

Rüppell said the Simien fox 'lives in packs and hunts tame sheep and small game'6, but most recent accounts refer to single animals or small groups; nobody has reported cooperative or pack hunting. Many observations confirm that the fox hunts by walking slowly through areas of high rodent density, pausing frequently to investigate a hole or tussock and cocking an ear as the colonial rodents give their high-pitched alarm calls. Despite turning aside and back tracking frequently most foxes when hunting seemed to travel in a more or less consistent direction, covering between 1 and $2 \mathrm{~km}$ in an hour. In the commonest method of capture the fox moved stealthily towards its prey, taking short tense steps in a crouched position, and freezing for anything up to 10 minutes, sometimes with its belly pressed flat to the ground, before dashing the last few metres to grab the quarry. Occasionally, mainly in longer grass where the prey was probably located by ear, they pounced, jack-knifing to bring the forepaws down in a stab. Only one chase was seen when a fox pursued an adult hare Lepus capensis for about $100 \mathrm{~m}$, steadily losing ground, before a second fox took over and, running flat out, came within $2 \mathrm{~m}$ of the quarry before being in turn outpaced after a further $300 \mathrm{~m}$. Occasionally they dig out their prey, the effort expended varying from a few half-hearted scratches at the mouth of a rat hole to the total destruction of a set of burrows leaving mounds of earth a metre high. One fox was seen digging out a rodent burrow to a depth of half-a-metre before extracting the occupant, and previous observers have recorded similar excavations. Only two out of 22 observed attempts to pounce on, dash at or chase prey were successful; a third animal was dug out and a fourth apparently picked up in passing. Of the four three were eaten on the spot and one cached. Brown ${ }^{4}$ recorded a fox finding a nest of young rats or fledgling birds.

\section{Diet}

Diurnal rodents, which are astonishingly numerous in the Bale Mountains, appeared to be the most likely prey. Many observers have watched foxes catch these small mammals without certainly identifying them. Recent collecting expeditions have established which species are present (including two new to science), and to obtain more precise and quantitative information on food we collected three batches of faeces, a relatively simple task on open ground:

A - from the Urgana Valley, south east of Dinshu, c. $3800 \mathrm{~m}$, January 1972.

B - from the Sanetti Plateau, April 1975

C - from the Sanetti Plateau, January/February 1976

both Afro alpine moorland
$3800-4,100 \mathrm{~m}$

A fourth batch collected around Gaysay Mountain, west of Dinshu, was excluded from our detailed analysis because at lower altitudes there is a danger of confusion with the droppings of other carnivores, e.g. jackal and serval; this does not apply on the high Sanetti plateau where there are few, if any, other mammalian carnivores, and faeces were collected from areas where foxes were frequently seen. The fox faeces were large (about $100 \mathrm{~mm}$ long $\times 35$ $\mathrm{mm}$ diameter). 
Table 1 Minimum numbers of species represented by teeth and bone fragments in Simien fox faeces from the high moorland of the Balé Mountains.

\begin{tabular}{|c|c|c|c|c|c|c|}
\hline & \multicolumn{3}{|c|}{ Sample } & \multirow{2}{*}{$\begin{array}{c}\text { Total } \\
\text { Min. No. }\end{array}$} & \multirow{2}{*}{$\begin{array}{c}\% \text { of } \\
\text { Total No }\end{array}$} \\
\hline & & $A^{*}$ & $B^{2}$ & $C$ & & \\
\hline \multirow{3}{*}{ Mole rats } & Tachyoryctes macrocephalus & 3 & 2 & 20 & 25 & $35 \cdot 2$ \\
\hline & Tachyoryctes splendens & 1 & 0 & 1 & 2 & $2 \cdot 8$ \\
\hline & Arvicanthis blicki & 0 & 0 & 13 & 13 & $18 \cdot 3$ \\
\hline \multirow{3}{*}{$\begin{array}{c}\text { Rat sized } \\
\text { rodents }\end{array}$} & Stenocephalemys & 2 & 4 & 1 & 7 & $9 \cdot 9$ \\
\hline & Lophuromys & 2 & 0 & 0 & 2 & $2 \cdot 8$ \\
\hline & Otomys typus & 13 & 0 & 1 & 14 & $19 \cdot 7$ \\
\hline Hare & Lepus capensis & 1 & 1 & 5 & 7 & 9.9 \\
\hline \multicolumn{2}{|c|}{ Very small mouse, unidentifiable } & 0 & 0 & 1 & $\begin{array}{r}1 \\
71\end{array}$ & $1 \cdot 4$ \\
\hline
\end{tabular}

* Sample A = Urgana Valley, $B+C=$ Sanetti Plateau, south of Goba

In samples $\mathrm{A}$ and $\mathrm{B}$, totalling about $720 \mathrm{~g}$ dry weight, the individual faecal pellets were aggregated, and from each sample a batch of teeth and bone fragments (weighing $74.5 \mathrm{~g}$ and $53 \mathrm{~g}$ respectively) was extracted. In sample C each pellet was treated separately, enabling a more exact estimate of numbers of prey to be made. Some of the bone fragments in the faeces were surprisingly large and sharp; several exceeded $30 \mathrm{~mm}$ in length. The largest piece $(36 \mathrm{~mm})$ was a lower incisor and part of the jaw from a mole rat; the intact palate and tooth rows of another mole rat weighed $1.4 \mathrm{~g}$. Other large pieces were from hares and included part of the ilium, incisor teeth, a proximal tibia epiphysis, and an auditory bulla. These suggest that, despite its unusually long jaws which one might presume to be rather weak, the Simien fox is still able to chew up the head and long bones of at least a medium-sized hare.

Some pellets contained remains from at least four prey items, but with such small samples of faeces, percentage occurrence of species indicates little more than which things are eaten most often (Table 1). To assess their relative nutritional importance in the diet, some account must be taken of the differing body weights: one hare is a larger meal than three rats. This is shown in Table 2 where the percentages shown represent the minimum number of animals eaten (Table 1) multiplied by a conversion factor (derived from trapping data) to give the relative weights of animals consumed. In the giant mole rat, Tachyoryctes macrocephalus, upper-incisor width is proportional to body weight ${ }^{13}$, a factor used to estimate the individual weights of those eaten $(100 \mathrm{~g}$ to $700 \mathrm{~g}$ ). The hares eaten varied considerably in size. One was a small leveret (perhaps only $500 \mathrm{~g}$ ), another was larger, though still young (having unfused epiphyses), but some may weigh $2 \mathrm{~kg}$ or more. Because of these disparities, and the probability that large hares would not be entirely consumed anyway, we have assigned a nominal body weight of $1 \mathrm{~kg}$ to the hares. Hares Lepus capensis and mole rats clearly formed the bulk of the prey ( 80 per cent by weight), though the smaller rodents were taken more often (comprising 52 per cent of the animals caught).

Hares were common on the alpine moors, with as many as 30 seen in a day's walking, but only one attempt by a fox to catch them was seen.

Mole rats belonged to two species which were often difficult to distinguish after chewing and digestion by a fox. The giant mole rat, despite its shyness, 
Table 2 Composition of Simien fox diet by weight

Minimum numbers of animals recorded from remains in faeces (Table 1) are multiplied by conversion factors based on average body weights of prey to estimate weight eaten of particular prey species. See text.

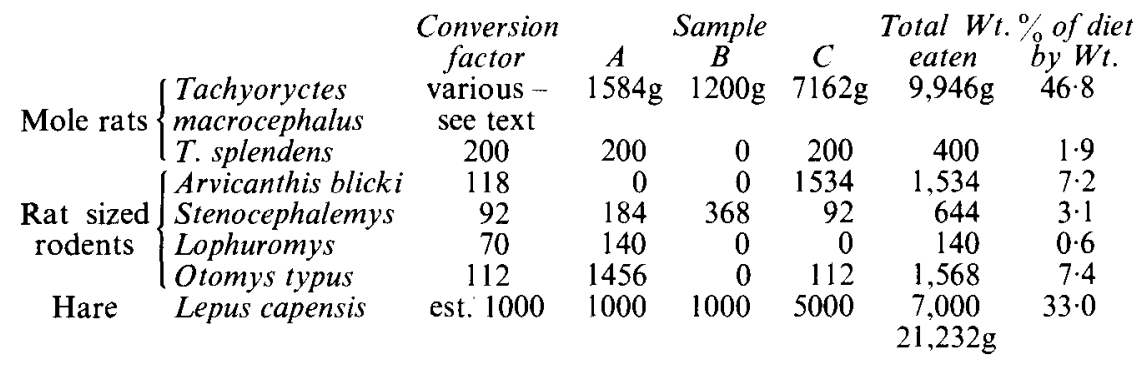

comprised 42 per cent of the diet by weight ( 32 per cent of the animals caught), and is thus by far the most important source of food for the Simien foxes in Balé. These curious creatures, restricted in range yet locally very abundant on the open moorland, spent only brief periods above ground collecting forage around their burrows and were extremely wary of any movement when on the surface ${ }^{13}$. They would occasionally emerge 2 or 3 metres from a stationary observer, and the fox's ability to freeze for long periods may be useful when hunting them. It is also possible that they are sometimes dug out. Adults were taken as well as youngsters. The common mole rat $T$. splendens, found widely in East Africa, was not important in the fox's diet on the high plateau (2.8 per cent of the animals caught), but the faeces from around Dinshu (possibly not all produced by foxes) revealed that it formed 32 per cent of all the animals eaten at the lower altitudes, where the giant mole rat was scarce or absent.

Rat-sized rodents: Otomys typus and Arvicanthis blicki are highland rodents of similar size. The former is common in long grass close to water; the latter, a colonial, diurnal species, is exceedingly abundant in the drier areas with shorter grass. Each forms 7 per cent of the fox's diet by weight, but 38 per cent of the animals caught. Almost all the Otomys came from the Urgana Valley, while Arvicanthis was the commonest rat caught on the Sanetti Plateau; it might have been more frequently predated, like the similarly diurnal giant mole rats, but for the significant advantage of living in colonies where the approach of an observer (and presumably a fox) is rapidly signalled by high-pitched squeaks until the danger is past. Lophuromys melanonyx, another common, endemic, diurnal rodent, was rare in the faeces, perhaps because it is small. It also lives in colonies which may afford some protection. Stenocephalemys, a locally endemic genus, and the commonest small mammal trapped at night, comprised only 3 per cent of the fox's diet (10 per cent of the animals caught). Its presence tends to confirm that the foxes are sometimes active after dark, but diurnal rodents are clearly more important fox food in Balé.

Our examination of the faecal contents was sufficiently exacting to reveal single rodent teeth and phalanges, but we found no trace of any shrews, despite their local abundance. Perhaps the large skin glands of the locally abundant Crocidura spp. rendered them distasteful to foxes, for they were the 


\section{Table 3 Groups observed in January and February}

\begin{tabular}{lcccccccc} 
& \multicolumn{7}{c}{ Group size } & \\
\cline { 2 - 8 } & 1 & 2 & 3 & 4 & 5 & 6 & 7 & Total of foxes \\
J.R.M. 1976 & 117 & 13 & 1 & 4 & 1 & 1 & 1 & 180 \\
L.H.Brown 1966 & 13 & 3 & 1 & 3 & 1 & 0 & 0 & 39
\end{tabular}

third most numerous item in the diet of an owl, Asio abyssinicus, in the Urgana Valley ${ }^{12}$. Among the faeces examined, we found the incisor teeth of one tiny unidentifiable mouse and also some claws possibly from a mole rat. Local people agree with Rüppell that the fox hunts 'tame sheep and small game', and human persecution is an important reason for the fox's decline in the Simien Mountains. However, Brown failed to find any trace of wool or long hairs in droppings collected in the Simien ${ }^{3}$, and we too found no evidence that domestic animals formed part of the Simien fox's diet. Even around Dinshu, where cattle and goats were abundant, there was no trace of them among any of the carnivore faeces collected, though at least 66 small mammals were represented by teeth, fur and fragments of bone.

\section{Social Behaviour}

Detailed behaviour study was hampered by the fact that individual animals were not distinguished, sexes could not be determined and it was difficult for an observer to remain undetected in the very open habitat. In a long-term study it may be possible to identify individuals by coat colour and markings. Groups of up to seven were seen, but 65 per cent (117/180) of sightings by J.R.M. were of solitary animals (Table 3). Only two pairs were seen on the Sanetti Plateau from January 9 th-16th; 10 pairs were seen from January 22nd-29th, of which three slept together.

Foxes seen in the middle of the day were almost always solitary, although the hunting ranges of different individuals appeared to overlap considerably, with up to four foxes visible at the same time hunting within an area of 2 square kilometres; only twice were animals seen to come together and each time they moved apart after a brief greeting in which they touched muzzles. Twice foxes lay down flat on the ground as another passed $70 \mathrm{~m}$ away as if to avoid detection or interference, and twice foxes resting between 100 and $200 \mathrm{~m}$ from a foraging animal kept a close eye on the hunter, even standing to look, but made no effort to approach. On another occasion, two foxes were resting only $20 \mathrm{~m}$ apart but no interaction was seen in an hour, after which one moved off alone.

Around dawn and dusk, when most of the foxes sighted were in groups, more elaborate and friendly social interactions were seen. Play involved chases and invitations to chase with one animal bounding up to within a few feet of another before dashing off. Mutual allogrooming was seen in one pair, and two pairs and a group of four were seen lying in contact. But the commonest and most striking interactions were the energetic and noisy greetings that seemed to occur primarily when groups formed, although one appeared to start spontaneously in a group of four before they moved off. Foxes were seen bounding about with tails waving and ears pressed, pushing 
their muzzles towards the faces of other foxes. The amount of noise produced was remarkable and clearly audible a kilometre away. Brown described the predominant vocalisation, which is similar to the 'yipes' of black-backed jackals Canis mesomelas but louder, as a 'long-drawn, high-pitched scream' rendered 'whee-ah whee-ah'. The call varied from an almost pure howl to a staccato scream interspersed with barks. In addition yips, whines and little 'wuf' barks were common.

Some foxes were attracted by the noise of greetings and the group of seven formed when 3 foxes joined 2 pairs that had come together with a loud greeting. Other foxes ignored the sounds of nearby greetings. No fox appeared to be foraging when in a group, but groups were seen trotting in open formation over the moorlands with a party of four being followed as they travelled $2 \mathrm{kms}$ in a consistent direction. Twice it appeared that solitary foxes moved away fast to avoid a group.

Some foxes were observed 'marking' prominent objects with urine, and the behaviour of others suggested that olfaction played an important role in communication and social behaviour. Urinating with a leg lifted was seen 18 times; in 15 the urine was directed at some emergent rock or shrub, and on another the fox scratched the ground after marking. Marking was seen three times in travelling groups: 2 foxes marked 3 times in an hour's foraging and another twice in half an hour. In areas of high fox density it appeared that droppings has been deposited in conspicuous places notably on the tops of the large mounds of earth beside the burrows of giant mole rats. Faeces were clustered in some places; 17 were counted in one area $5 \mathrm{~m}$ by $6 \mathrm{~m}$.

No direct evidence of territoriality was observed but the movement and marking of foxes in groups and the noise generated in greetings are all suggestive, although some animals apparently did not take part in these activities. One fox that had evaded a group was followed for 4 hours during which it squatted to urinate once but was not seen to mark. Pairs appeared to be forming in late January 1976; at other times of year and at lower densities the social system may differ considerably.

\section{Reproduction}

Nothing is known about the reproductive cycle in Simien foxes. Young pups have never been reported from the Bale Mountains, and in January and February older ones could not be confidently distinguished from adults, suggesting that births do not occur later than May or June. However, Tyler (1975) reported an assumed adult and two well grown cubs lying together on the Sanetti Plateau in May, suggesting an overlap in the cubs of successive years. Perhaps the female only separates from her family when a new litter is born? On one occasion four foxes in a group of five lay in contact, which is characteristic of litter-mates among many canids, and suggests that the Simien fox may produce up to four young in a litter.

\section{Response to Humans}

Response to observers was variable, but out of 66 occasions when foxes were aware of the observer, they gave alarm calls 45 times, 68 per cent. The alarm call is a loud, repeated screeching noise rendered by Brown as 'yeahp, yeahp' ${ }^{3}$. On three occasions an alarm call appeared to elicit a greeting from nearby foxes, but on six others a distant alarm call brought no audible response. In 
general the Bale foxes are wary but not shy. They would yelp loudly on sighting us, then appear unconcerned and resume normal activity, sometimes as near as 100 metres but usually $200 \mathrm{~m}$ or more away. They showed little or no fear of motor vehicles and could be approached to within $15-20 \mathrm{~m}$ without apparently disrupting their normal behaviour.

\section{Conclusion}

The Simien fox is an interesting and integral part of Ethiopia's high-altitude mammal fauna, predating (probably exclusively) other montane mammals and not domestic stock. Unlike most canids, it seems to thrive in open country above $3000 \mathrm{~m}$, where rodent densities are very high, but its restriction to limited habitats and only in Ethiopia means it has probably never been abundant. Of the four remaining known populations, three are close to extinction - in the Simien Mountains, north-west Shoa, and the Arussi Mountains. The main cause of decline is the increase in agriculture at high altitudes, accelerated in the Simien, and probably elsewhere, by direct persecution in the unfounded belief that foxes kill sheep and because their liver is used as a medicament ${ }^{10}$.

The Balé Mountains almost certainly support the last large population, probably numbering over 400 , of which at least 350 live within a proposed national park. The areas with the most foxes are sufficiently high and inhospitable to have deterred much human interference to date, and the local Galla tribe appear to tolerate them. In January 1976 a healthy population of foxes, with 10 seen in one morning, were coexisting with about 2000 cattle in the Ueb Valley in the west of the proposed park. But their status will remain uncertain until the national park has been properly gazetted. A new hazard was added when a road across the Sanetti Plateau, the area of highest fox density, was recently completed, from which the foxes are now easily seen and where they are exposed to persecution because of their fearlessness of motor vehicles. However, if the national park can be properly protected, and the present levels of grazing and habitat destruction there are not increased, the Simien fox should continue to survive, if not prosper.

\section{Acknowledgments}

Dr D. W. Yalden painstakingly checked the identity of all the material used in this study, and the authors would also like to thank him and G. I. Reid for their help in the field. The Royal Society, the Percy Sladen Memorial Trust and the Godman Fund generously provided financial assistance for both expeditions to the Balé Mountains by P.A.M. The work of J.R.M. was greatly facilitated by the assistance of J. G. Stephenson and Ato Abbai of the Ethiopian Wildlife Conservation Organisation, and supported by a grant from the Fauna Preservation Society.

\section{References}

1. ANON (1974) Oryx 12542.

2. BOLTON, M. 1973 Notes on the current status and distribution of some large mammals in Ethiopia. Mammalia 37 562-586.

3. BROWN, L. 1964 Simien Fox. Africana 2 45-48.

4. BROWN, L.H. 1966 A report on the National Geographic Society/World Wildlife Fund Expedition to study the Mountain Nyala (Tragelaphus buxtoni).

5. CLUTTON-BROCK, J., CORBET, G.B. \& HILLS, M. 1976 A review of the family Canidae, with a classification by numerical methods. Bull. Brit. mus. (nat. Hist.) Zool 29 117-199. 
6. HARPER, F. 1945 Extinct and vanishing mammals of the old world. Special Publication 12. American Committee for International Wildlife Protection. New York.

7. IUCN 1972 Red Data Book: Mammalia. IUCN, Morges, Switzerland.

8. MAYDON, H.C. ed. 1932 Big game shooting in Africa. London.

9. POWELL-COTTON, P.H.G. 1902 A sporting trip through Abyssinia. London.

10. STAEHL 1975 Report on Simien Mountains National Park. In World Wildlife Fund Yearbook 1974-5. Morges, Switzerland.

11. TYLER, S. 1975 The Simien Fox. Wildlife 17 564-5.

12. YALDEN, D.W. 1973 Prey of the Abyssinian long-eared owl, Asio abyssinicus. Ibis 115 605-606.

13. YALDEN, D.W. 1975 Some observations on the giant mole rat Tachyoryctes macrocephalus (Rüppell, 1842) (Mammalia, Rhizomyidae) of Ethiopia. Mon. zool. ital. 15 275-303.

Dr Patrick A. Morris, Royal Holloway College, Englefeld Green, Surrey TW20 9TY.

\section{Man Pressure Again}

\section{G. C. L. Bertram}

In 1963 Oryx published an article by Dr Bertram called Man Pressure. This second article returns to the theme, the argument being now reinforced by the addition in the interval of another 1000 million people to the world's population. At this rate what hope have we of conserving the other species that share the planet, and without them what hope is there for human survival?

'Man Pressure' described the basic and overwhelming pressure of ourselves upon all that great variety of animal and plant species, the glory of our planet, which we seek to preserve and conserve for an extended future. Since then, in a mere fourteen years, almost 1000 million more people have been added to the world in competition with those other species which we use, enjoy and hold in trust. Our species now numbers over 4000 million, all aspirants to a sufficiency of food and a level of material well-being far higher than all but the relatively few already enjoy (and some squander to excess). Our population explosion is like nuclear warfare in that the enormity of each is so great that many seek to forget the consequences, and the two are connected because in some degree the fervour generated by the former increases the probability of the latter, and the second may well finally 'solve' the first. But both enormities are still within our control if we will but exert ourselves sufficiently and rationally. If we do not, all that we hold dear in quality of life, and even in life itself may end. Decades of great danger are inevitable but the possibility of success must spur us on.

The fifth billion of human kind will take only another eleven years to arrive and we shall be over 6000 million by the turn of the century and still vastly proliferating. Robert S. McNamara, President of the World Bank, a realist but one not without hope of ultimate success, has recently provided us with an excellent conspectus of human demographic affairs. So important is the issue and succinct his summary that I quote: 'It now appears that a significant decline in fertility may have at last begun in the developing countries. The data are not yet fully conclusive ... (but) ... if . . continued ... then what we are seeing here is something of historic importance. It would mean that the period of rapid acceleration in the rate of growth of the world's population has finally 